\title{
From High-level Component-Based Models to Distributed Implementations
}

\author{
Borzoo Bonakdarpour \\ Marius Bozga \\ Mohamad Jaber \\ Jean Quilbeuf \\ Joseph Sifakis
}

VERIMAG, 2 avenue de Vignate, 38610, GIÈRES, France

\begin{abstract}
Although distributed systems are widely used nowadays, their implementation and deployment is still a time-consuming, error-prone, and hardly predictive task. In this paper, we propose a methodology for producing automatically efficient and correct-by-construction distributed implementations by starting from a high-level model of the application software in BIP. BIP (Behavior, Interaction, Priority) is a componentbased framework with formal semantics that rely on multiparty interactions for synchronizing components and dynamic priorities for scheduling between interactions.

Our methodology transforms arbitrary BIP models into Send/Receive BIP models, directly implementable on distributed execution platforms. The transformation consists of (1) breaking atomicity of actions in atomic components by replacing strong synchronizations with asynchronous Send/Receive interactions; (2) inserting several distributed controllers that coordinate execution of interactions according to a user-defined partition, and (3) augmenting the model with a distributed algorithm for handling conflicts between controllers. The obtained Send/Receive BIP models are proven observationally equivalent to the initial models. Hence, all the functional properties are preserved by construction in the implementation. Moreover, Send/Receive BIP models can be used to automatically derive distributed implementations. Currently, it is possible to generate stand-alone $\mathrm{C}++$ implementations using either TCP sockets for conventional communication, or MPI implementation, for deployment on multicore platforms. This method is fully implemented. We report concrete results obtained under different scenarios (i.e., partitioning of the interactions and choice of algorithm for distributed conflict resolution).
\end{abstract}

\section{Keywords}

Component-based modeling, Automated transformation, Distributed systems, BIP, Correctness-by-construction, Committee coordination, Conflict resolution.

\section{INTRODUCTION}

Analysis and design of computing systems often starts with developing a high-level model of the system. Constructing models is beneficial, as designers can abstract away implementation details and validate the model with respect to a set of intended requirements through different techniques such as formal verification, simulation, and testing. However, deriving a correct implementation from a model is always challenging, since adding implementation details involves many

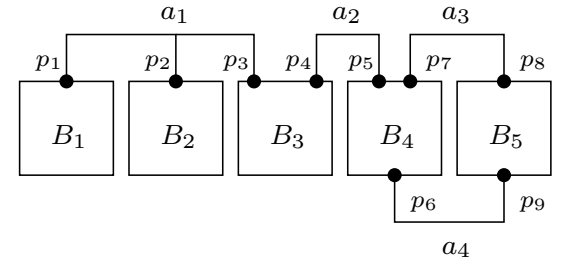

Figure 1: A simple BIP model with conflicts.

subtleties that can potentially introduce errors to the resulting system. In the context of distributed systems, these subtleties are amplified significantly because of inherently concurrent, non-deterministic, and non-atomic structure of distributed systems, as well as the occurrence of unanticipated physical and computational events such as faults. Thus, it is highly advantageous if designers can somehow derive implementations in a systematic and ideally automated correct fashion from high-level models. It is, nonetheless, unclear how to transform an abstract model (where atomicity is assumed through global state semantics and distribution details are omitted via employing high-level synchronization primitives) into a real distributed implementation.

In this paper, we present a novel method for automatically transforming high-level models in BIP [?] into distributed implementations. The BIP (Behavior, Interaction, Priority) language is based on a semantic model encompassing composition of heterogeneous components. The behavior of atomic components is described as an automaton or Petri net extended by data and functions given in $\mathrm{C}++$. Transitions of the automaton or Petri net are labeled by port names and functions computing data transformations when they are executed. If a transition of the Petri net can be executed, we say that the associated port is enabled. BIP uses a composition operator for obtaining composite components from a set of atomic components. The operator is parameterized by a set of interactions between the composed components. One such interaction type is rendezvous, which is enabled if all of its participating ports are enabled. Currently, BIP has a formal operational semantics. The execution of interactions and local code of components are orchestrated by a sequential scheduler.

In order to understand the subtleties of transforming a BIP model into a distributed implementation, consider the BIP model in Figure 1. In this model, atomic components $B_{1} \cdots B_{5}$ are synchronized by four rendezvous interactions $a_{1} \cdots a_{4}$. In sequential models, interactions are executed atomically by a single scheduler. To the contrary, introducing concurrency and distribution to this model requires the imple- 
mentation to deal with more complex issues:

- (Partial observability) Suppose interaction $a_{1}$ (and, hence, components $B_{1} \cdots B_{3}$ ) is being executed. If component $B_{3}$ completes its computation before $B_{1}$ and $B_{2}$, and, ports $p_{4}$ and $p_{5}$ are enabled, then interaction $a_{2}$ is enabled. In such a case, a distributed scheduler must be designed, so that concurrent execution of interactions does not introduce behaviors that were not allowed by the high-level model.

- (Resolving conflicts) Suppose interactions $a_{1}$ and $a_{2}$ are enabled simultaneously. Since these interactions share component $B_{3}$, they cannot be executed concurrently. We call such interactions conflicting. Obviously, a distributed scheduler must ensure that execution of conflicting interactions is mutually exclusive.

- (Performance) On top of correctness issues, a real challenge is to ensure that a transformation does not add considerable overhead to the implementation. After all, one crucial goal of developing distributed and parallel systems is to exploit their computing power.

We address the issue of partial observability by breaking the atomicity of execution of interactions, so that a component can execute unobservable actions once the corresponding interaction is being executed [3]. Resolving conflicts leads us to solving the committee coordination problem [5], where a set of professors organize themselves in different committees and two committees that have a professor in common cannot meet simultaneously. The original distributed solution to the committee coordination problem assigns one manager to each interaction [5]. Conflicts between interactions are resolved by reducing the problem to the dining or drinking philosophers problems [?], where each manager is mapped onto a philosopher. Bagrodia [1] proposes an algorithm where message counts are used to solve synchronization and exclusion is ensured by using a circulating token. In a follow-up paper [2], Bagrodia modifies the solution in [1] by using message counts to ensure synchronization and reducing the conflict resolution problem to dining or drinking philosophers. Also, Perez et al [?] propose another approach that essentially implements the same idea using a lock-based synchronization mechanism.

As Bagrodia notes [2], a family of solutions to the committee coordination problem is possible, ranging over fully centralized to fully decentralized ones, depending upon the mapping of sets of committees to the managers. Thus, augmenting a transformation with different families of solutions results in different distributed implementations of the initial BIP model. We expect that each class of solutions exhibits advantages and disadvantages and, hence, fits a specific type of applications on a target architecture and platform. Although the algorithms in $[1,2,5]$ provide us with different families of solutions, transforming a high-level model into a concrete distributed implementation involves other details that have not been taken into account. Examples include preserving functional properties of the original model, computations associated with interactions and components, data transfer, level of concurrency, fairness, fault-tolerance, efficiency, and performance. These issues can significantly change the dynamics and performance of a distributed implementation and each deserves rigorous research beyond the algorithms and preliminary simulations in $[1,2,5]$. We believe we currently lack a deep understanding of the impact of these issues and their correlation in transforming high-level models into concrete distributed implementations.
Contributions. With this motivation, in this paper, we propose a generic framework for transforming high-level BIP models into a distributed implementation that allow parallelism between components as well as parallel execution of non-conflicting interactions by embedding a solution to the committee coordination problem. To the best of our knowledge, this is the first instance of such a transformation (the related work mentioned above only focus on impossibility results, abstract algorithms, and in one instance [2] simulation of an algorithm). Our method utilizes the following sequence of transformations preserving observational equivalence:

1. First, we transform the given BIP model into another BIP model that (1) operates in partial-state semantics, and (2) expresses multi-party interactions in terms of asynchronous message passing (Send/Receive primitives). Moreover, the target BIP model is structured in three layers:

(a) The components layer consists of a transformation of behavioral components in the original model.

(b) The interaction protocol detects enabledness of interactions of the original model and executes them after resolving conflicts either locally or by the help of the third layer. This layer consists of a set of components, each hosting a user-defined subset of interactions from the original BIP model.

(c) The reservation protocol resolves conflicts requested by the interaction protocol. The reservation protocol implements a committee coordination algorithm and our design allows employing any such algorithm. We, in particular, consider three committee coordination algorithms: (1) a fully centralized algorithm, (2) a token-based distributed algorithm, and (3) an algorithm based on reduction to distributed dining philosophers.

2. Then, we transform the 3-layer BIP model into $\mathrm{C}++$ code that employs TCP sockets for communication.

We also conduct a set of experiments to analyze the behavior and performance of the generated code using different scenarios (i.e., different partitioning schemes and choice of committee coordination algorithm). Our experiments clearly show that each scenario is suitable for a different topology, size of the distributed system, communication load, and of course, the structure of the initial BIP model.

Organization. In Section 2, we present the global state operational semantics of BIP. We describe our 3-layer model in Section 3. Section 4 is dedicated to detailed description of our BIP to BIP transformation. In Section 5, we show the correctness of our transformation. Section 6 presents the results of our experiments. Finally, in Section 7, we make concluding remarks and discuss future work.

\section{BASIC SEMANTIC MODELS OF BIP}

In this section, we present operational global state semantics of BIP. BIP is a component framework for constructing systems by superposing three layers of modeling: Behavior, Interaction, and Priority. Since the issue of priorities is irrelevant to this paper, we omit it.

Atomic Components We define atomic components as transition systems with a set of ports labeling individual transitions. These ports are used for communication between different components. 
Definition 1 (Atomic Component) An atomic component $B$ is a labeled transition system represented by a triple $(Q, P, \rightarrow$ ) where $Q$ is a set of states, $P$ is a set of communication ports, $\rightarrow \subseteq Q \times P \times Q$ is a set of possible transitions, each labeled by some port.

For any pair of states $q, q^{\prime} \in Q$ and a port $p \in P$, we write $q \stackrel{p}{\rightarrow} q^{\prime}$, iff $\left(q, p, q^{\prime}\right) \in \rightarrow$. When the communication port is irrelevant, we simply write $q \rightarrow q^{\prime}$. Similarly, $q \stackrel{p}{\rightarrow}$ means that there exists $q^{\prime} \in Q$ such that $q \stackrel{p}{\rightarrow} q^{\prime}$. In this case, we say that $p$ is enabled in state $q$.

In practice, atomic components are extended with variables. Each variable may be bound to a port and modified through interactions involving this port. We also associate a guard and an update function to each transition. A guard is a predicate on variables that must be true to allow the execution of the transition. An update function is a local computation triggered by the transition that modifies the variables. Figure 2(a) shows an atomic component $B$, where $Q=\{s, t\}$, $P=\{p, q, r\}$, and $\rightarrow=\{(s, p, t),(t, q, s),(t, r, t)\}$.

Interaction For a given system built from a set of $n$ atomic components $\left\{B_{i}=\left(Q_{i}, P_{i}, \rightarrow_{i}\right)\right\}_{i=1}^{n}$, we assume that their respective sets of ports are pairwise disjoint, i.e., for any two $i \neq j$ from $\{1 . . n\}$, we have $P_{i} \cap P_{j}=\emptyset$. We can therefore define the set $P=\bigcup_{i=1}^{n} P_{i}$ of all ports in the system. An $i n$ teraction is a set $a \subseteq P$ of ports. When we write $a=\left\{p_{i}\right\}_{i \in I}$, we suppose that for $i \in I, p_{i} \in P_{i}$, where $I \subseteq\{1 . . n\}$.

Similar to atomic components, BIP extends interactions by associating a guard and a transfer function to each of them. Both the guard and the function are defined over the variables that are bound to the ports of the interaction. The guard must be true to allow the interaction. When the interaction takes place, the associated transfer function is called and modifies the variables.

Definition 2 (Composite Component) A composite component (or simply component) is defined by a composition operator parameterized by a set of interactions $\gamma \subseteq 2^{P} . B \stackrel{\text { def }}{=}$ $\gamma\left(B_{1}, \ldots, B_{n}\right)$, is a transition system $(Q, \gamma, \rightarrow)$, where $Q=$ $\bigotimes_{i=1}^{n} Q_{i}$ and $\rightarrow$ is the least set of transitions satisfying the rule

$$
\frac{a=\left\{p_{i}\right\}_{i \in I} \in \gamma \quad \forall i \in I . q_{i}{\stackrel{p_{i}}{\rightarrow}}_{i} q_{i}^{\prime} \quad \forall i \notin I . q_{i}=q_{i}^{\prime}}{\left(q_{1}, \ldots, q_{n}\right) \stackrel{a}{\rightarrow}\left(q_{1}^{\prime}, \ldots, q_{n}^{\prime}\right)}
$$

The inference rule says that a composite component $B=$ $\gamma\left(B_{1}, \ldots, B_{n}\right)$ can execute an interaction $a \in \gamma$, iff for each port $p_{i} \in a$, the corresponding atomic component $B_{i}$ can execute a transition labeled with $p_{i}$; the states of components that do not participate in the interaction stay unchanged. Figure 2 (b) illustrates a composite component $\gamma\left(B_{0}, B_{1}\right)$, where each $B_{i}$ is identical to component $B$ in Figure 2(a) and $\gamma=$ $\left\{\left\{p_{0}, p_{1}\right\},\left\{r_{0}, r_{1}\right\},\left\{q_{0}\right\},\left\{q_{1}\right\}\right\}$.

\section{THE 3-LAYER ARCHITECTURE}

In this section, we describe the overall architecture of our BIP source-to-source transformation. Since we target a distributed setting, we assume concurrent execution of interactions. However, if two interactions are simultaneously enabled, they cannot always run in parallel without breaking

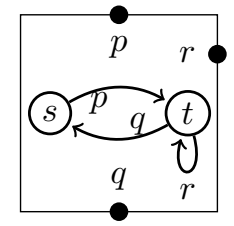

(a) Atomic component

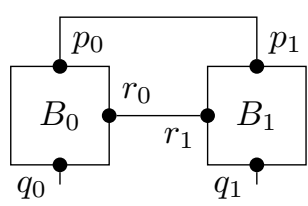

(b) Composite component

\section{Figure 2: BIP composite component}

semantics of the global state model. This leads to the notion of structural conflicts between interactions.

Definition 3 Let $\gamma\left(B_{1}, \ldots, B_{n}\right)$ be a BIP model. We say that two interactions $a_{1}, a_{2} \in \gamma$ are conflicting iff either:

- they share a common port $p$; i.e., $p \in a_{1} \cap a_{2}$, or

- there exist an atomic component $B_{i}=\left(Q_{i}, P_{i}, \rightarrow_{i}\right)$, a state $q \in Q_{i}$, and two ports $p_{1}, p_{2} \in P_{i}$ such that (1) $p_{1} \in a_{1}$, (2) $p_{2} \in a_{2}$, and (3) $q \stackrel{p_{1}}{\longrightarrow} \wedge q \stackrel{p_{2}}{\longrightarrow}$.

As discussed in the introduction, handling conflicting interactions in a BIP model running by a sequential scheduler is quite straightforward. However, in a distributed setting, detecting and avoiding conflicts are not trivial. Thus, our target BIP model in a transformation should have the following three properties: (1) preserving the behavior of each atomic component, (2) preserving the observational behavior of interactions, and (3) resolving conflicts in a distributed manner. Since several distributed algorithms exist in the literature for conflict resolution, we design our framework, so that it provides appropriate interfaces with minimal restrictions. Moreover, we require that interactions in the target model are of the form Send/Receive with one sender and multiple receivers. Such interactions can be implemented using conventional communication primitives (e.g., TCP sockets or MPI).

Definition 4 We say that $B^{S R}=\gamma^{S R}\left(B_{1}^{S R}, \ldots, B_{n}^{S R}\right)$ is a Send/Receive BIP composite component iff we can partition the set of ports in $B^{S R}$ into three sets $P_{s}, P_{r}, P_{u}$ that are respectively the set of send-ports, receive-ports, and unary interaction ports, such that:

- Each interaction $a \in \gamma^{S R}$, is either a Send/Receive interaction $a=\left(s, r_{1}, r_{2}, \ldots, r_{k}\right)$ with $s \in P_{s}$ and $r_{i} \in P_{r}$, or, a unary interaction $a=\{p\}$ with $p \in P_{u}$.

- If $s$ is a port in $P_{s}$, then there exists one and only one Send/Receive interaction $\left(s, r_{1}, r_{2}, \ldots, r_{k}\right) \in \gamma^{S R}$ where all ports $r_{1}, \ldots, r_{k}$ are receive-ports. We say that $r_{1}, r_{2}, \ldots, r_{k}$ are the receive-ports associated to $s$.

- If $\left(s, r_{1}, \ldots, r_{k}\right)$ is a Send/Receive interaction in $\gamma^{S R}$ and $s$ is enabled at some global state of $B^{S R}$, then all its associated receive-ports $r_{1}, \ldots, r_{k}$ are also enabled at that state.

We design our target BIP model based on the three tasks identified above, where we incorporate one layer for each task. We use the high-level BIP model in Figure 1 as a running example throughout the paper to describe the concepts of our transformation. We assume that interaction $a_{1}$ is in conflict with only interaction $a_{2}$, and, interactions $a_{2}, a_{3}$, and $a_{4}$ are in pairwise conflict. Our 3-layer architecture consists of the following layers. 


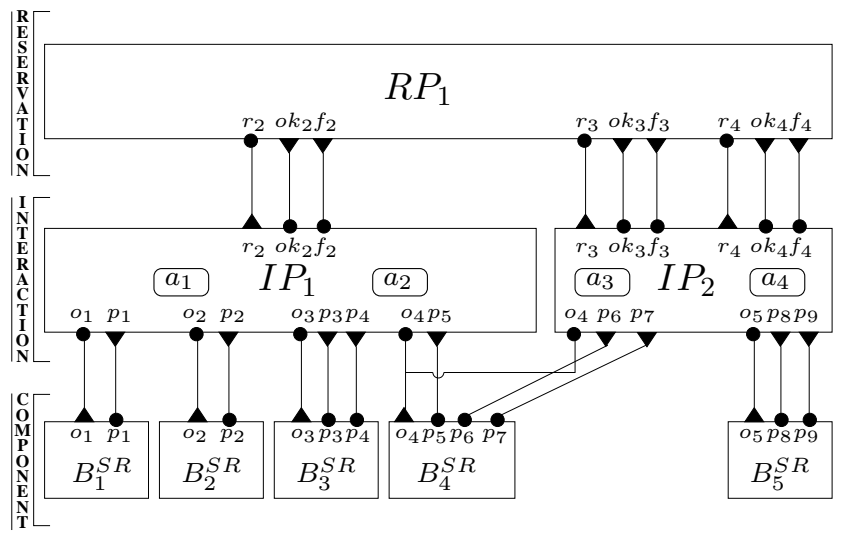

Figure 3: 3-layer model of Figure 1.

Components Layer. Atomic components in the high-level model are placed in this layer with the following additional ports per component. The send-port $o$ that shares the list of enabled ports in the component with the upper layer. Also, for each port $p$ in the original component, we include a receiveport $p$ through which the component is notified to execute the transition labeled by $p$ once the upper layers resolve conflicts and decide on which components can execute on what port. The bottom layer in Figure 3 includes components illustrated in Figure 1.

Interaction Protocol. This layer consists of a set of components each hosting a set of interactions in the high-level model. Conflicts between interactions included in the same component are resolved by that component locally. For instance, interactions $a_{1}$ and $a_{2}$ (resp. $a_{3}$ and $a_{4}$ ) of Figure 1 are grouped into component $I P_{1}$ (resp. component $\left.I P_{2}\right)$ in Figure 3. Thus, the conflict between $a_{1}$ and $a_{2}$ (resp. $a_{3}$ and $\left.a_{4}\right)$ is handled locally in $I P_{1}$ (resp. $\left.I P_{2}\right)$. To the contrary, the conflicts between $a_{2}$ and either $a_{3}$ or $a_{4}$ have to be resolved using an external algorithm that solves the committee coordination problem. Such an algorithm forms the top layer of our model. The interaction protocol also evaluates the guard of each interaction and executes the code associated with an interaction that is selected locally or by the upper layer. The interface between this layer and the component layer provides ports for receiving enabled ports from each component (i.e., port $o$ ) and notifying the components on permitted port for execution.

Reservation Protocol. This layer accommodates an algorithm that solves the committee coordination problem. For instance, the external conflicts between interactions $a_{2}$ and $a_{3}$, and, interactions $a_{2}$ and $a_{4}$ are resolved by the central component $R P_{1}$ in Figure 3. We emphasize that the structure of components in this layer solely depends upon the augmented conflict resolution algorithm. Incorporating a centralized algorithm results in one component $R P_{1}$ as illustrated in Figure 3. Other algorithms (as will be discussed in Subsection 4.3 ), such as ones that use a circulating token [1] or dining philosophers $[2,5]$ result in different structures. The interface between this layer and the Interaction Protocol involves ports for receiving request to reserve an interaction (labeled $r$ ) and responding by either success (labeled $o k$ ) or failure (labeled $f)$.

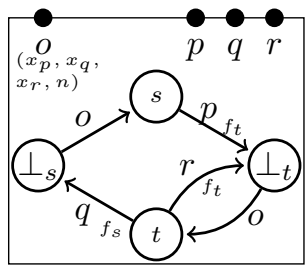

$$
\begin{gathered}
f_{s}=\left\{\begin{array}{l}
x_{p}:=\text { true } \\
x_{q}:=\text { false } \\
x_{r}:=\text { false } \\
n++
\end{array}\right. \\
f_{t}=\left\{\begin{array}{l}
x_{p}:=\text { false } \\
x_{q}:=\text { true } \\
x_{r}:=\text { true } \\
n++
\end{array}\right.
\end{gathered}
$$

Figure 4: SR atomic component of Figure 2(a).

\section{TRANSFORMING BIP INTO 3-LAYER BIP}

In this section, we describe our technique for transforming a high-level BIP model into a 3-layer BIP model in detail. Construction of the three layers are described in Subsections $4.1,4.2$, and 4.3 respectively. Finally, we describe cross-layer interactions in Subsection 4.4.

\subsection{Transformation of Atomic Components}

We now present how we transform an atomic component $B$ from a given BIP model into a Send/Receive atomic component $B^{S R}$ that is capable of communicating with the Interaction Protocol in the 3-layer model. As mentioned in Section $3, B^{S R}$ sends offers to the Interaction Protocol that are acknowledged by a response. An offer includes the set of enabled ports of $B^{S R}$ at the current state through which the component is ready to interact. Enabled ports are specified by a set of Boolean variables. These variables are modified by a port update function. The function evaluates each variable when reaching a new state. When the upper layers select an interaction involving $B^{S R}$ for execution, $B^{S R}$ is notified by a response sent on the port chosen. We also include a participation number variable $n$ in $B^{S R}$, which counts the number of interactions $B^{S R}$ has participated in.

Since each response triggers an internal computation, following [3], we split each state $s$ into two states, namely, $s$ itself and a busy state $\perp_{s}$. Intuitively, reaching $\perp_{s}$ marks the beginning of an unobservable internal computation. We are now ready to define the transformation from $B$ into $B^{S R}$.

Definition 5 Let $B=(Q, P, \rightarrow)$ be an atomic component. The corresponding Send/Receive atomic component is $B^{S R}=$ $\left(Q^{S R}, P^{S R}, \rightarrow^{S R}\right)$ with the additional variables $X$, such that:

- $Q^{S R}=Q \cup Q^{\perp}$, where $Q^{\perp}=\left\{\perp_{s} \mid s \in Q\right\}$.

- $P^{S R}=P \cup\{o\}$, where the set of variables $X=$ $\left\{x_{p}\right\}_{p \in P} \cup\{n\}$ are associated to offer port o. The port update function $f_{t}$ modifies $X$ as follows: it sets $x_{p}$ to true if $t \stackrel{p}{\longrightarrow}$, to false otherwise, and increments $n$.

- For each transition $(s, p, t) \in \rightarrow$, we include the following two transitions in $\rightarrow^{S R}:\left(\perp_{s}, o, s\right)$ and $\left(s, p, \perp_{t}\right)$. The transition $\left(s, p, \perp_{t}\right)$ triggers the function $f_{t}$.

Figure 4 illustrates transformation of the component in Figure 2(a) into its corresponding Send/Receive component.

\subsection{Interaction Protocol}

Given a high-level BIP model $B=\gamma\left(B_{0} \cdots B_{n}\right)$, one parameter to our transformation is a partition of interactions $\gamma_{1}, \ldots, \gamma_{m}$. Partitioning of interactions is a means for the designer to enforce load-balancing and improving the performance of the given model when running in a distributed fashion. It also determines whether or not a conflict between interactions can be resolved locally. We associate each class $\gamma_{j}$ of interactions to an Interaction Protocol component $I P_{j}$ 

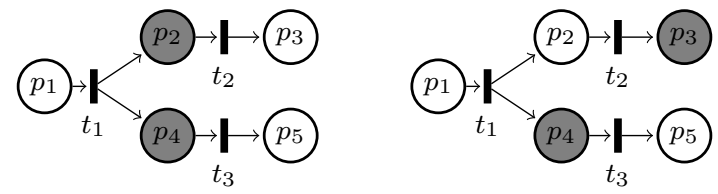

Figure 5: A simple Petri net

that is responsible for (1) detecting enabledness by collecting offers from the Components Layer, (2) selecting a set of nonconflicting interactions (either locally or by the help of the Reservation Protocol), and (3) executing the selected interactions in $\gamma_{i}$ by notifying the corresponding atomic components. For instance, in Figure 3, we have two classes: $\gamma_{1}=\left\{a_{1}, a_{2}\right\}$ (hosted by component $I P_{1}$ ) and $\gamma_{2}=\left\{a_{3}, a_{4}\right\}$ (hosted by component $\left.I P_{2}\right)$. In this section, for simplicity of reasoning about correctness, we construct the behavior of an IP component by a Petri net.

Definition 6 A 1-Safe Petri net is defined by a triple $S=$ $(L, P, T)$ where $L$ is a set of places, $P$ is a set of ports, and $T \subseteq 2^{L} \times P \times 2^{L}$ is a set of transitions. A transition $\tau$ is a triple $\left({ }^{\bullet} \tau, p, \tau^{\bullet}\right)$, where ${ }^{\bullet} \tau$ is the set of input places of $\tau$ and $\tau^{\bullet}$ is the set of output places of $\tau$.

We represent a Petri net as an oriented bipartite graph $G=(L \cup T, E)$. Places are represented by circular vertices and transitions are represented by rectangular vertices. The set of oriented edges $E$ is the union of the edges $\{(l, \tau) \in$ $\left.L \times T \mid l \in{ }^{\bullet} \tau\right\}$ and the edges $\left\{(\tau, l) \in T \times L \mid l \in \tau^{\bullet}\right\}$.

We depict the state of a Petri net by marking its places with tokens. We say that a place is marked if it contains a token. A transition $\tau$ can be executed if all its input places in - $\tau$ contain a token. Upon the execution of $\tau$, tokens in input places ${ }^{\bullet} \tau$ are removed and output places in $\tau^{\bullet}$ are marked. Formally, let $\longrightarrow_{S}$ be the set of triples $\left(m, p, m^{\prime}\right)$, such that $\exists \tau=\left({ }^{\bullet} \tau, p, \tau^{\bullet}\right) \in T$, where ${ }^{\bullet} \tau \subseteq m$ and $m^{\prime}=\left(m \backslash{ }^{\bullet} \tau\right) \cup \tau^{\bullet}$. The behavior of a Petri net $S$ can be defined by a labeled transition system $\left(2^{L}, P, \longrightarrow S\right)$.

Figure 5 shows an example of a Petri net in two successive markings. It has five places $\left\{p_{1}, \ldots, p_{5}\right\}$ and three transitions $\left\{t_{1}, t_{2}, t_{3}\right\}$. The places containing a token are depicted with gray background. The left Petri net is obtained by executing transition $t_{1}$. The right Petri net shows the resulting state of the left Petri net when transition $t_{2}$ is fired.

Since components of the interaction protocol deal with interactions of the original model, they need to be aware of conflicts in the original model as defined in Definition 3. We distinguish two types of conflicting interactions according to a given partition:

- External: two interactions are externally conflicting if they conflict and they belong to different classes of the partition. External conflicts are referred to the Reservation Protocol. For instance, in Figure 3, interaction $a_{2}$ is in external conflict with interactions $a_{3}$ and $a_{4}$.

- Internal: two interactions are internally conflicting if they conflict, but they belong to the same class of the partition. Internal conflicts are resolved by the Interaction Protocol within the component that hosts them. For instance, in Figure 3, interaction $a_{1}$ is in internal conflict with interaction $a_{2}$. If component $I P_{1}$ chooses interaction $a_{1}$ over $a_{2}$, no further action is required. Note, however, that if $I P_{1}$ chooses $a_{2}$, then it has to request its reservation from $R P_{1}$, as it is in conflict with $a_{3}$ and $a_{4}$ externally.
The Petri net that defines the behavior of an Interaction Protocol component $I P_{j}$ handling a class $\gamma_{j}$ of interactions is constructed as follows. We refer to Figure 6 as a concrete example for construction of the Petri net of component $I P_{1}$ in Figure 3.

Places. We include three types of places:

- For each component $B_{i}$ involved in interactions of $\gamma_{j}$, we include waiting and received places $w_{i}$ and $r c v_{i}$, respectively. $I P_{j}$ waits in a waiting place until it receives an offer from the corresponding component. When an offer from component $B_{i}$ is received (along with the fresh values of the Boolean variables associated to the ports of the sender), $I P_{j}$ moves from $w_{i}$ to $r c v_{i}$. In Figure 6 , since components $B_{1} \cdots B_{4}$ are involved in interactions hosted by $I P_{1}$ (i.e., $a_{1}$ and $a_{2}$ ), we include waiting places $w_{1} \cdots w_{4}$ and received places $r c v_{1} \cdots r c v_{4}$.

- For each port $p$ involved in interactions of $\gamma_{j}$, we include a sending place $s n d_{p}$. The response to an offer with $x_{p}=$ true is sent from this place to port $p$ of the component that has made the offer. In Figure 6, places $s n d_{p_{1}} \cdots s n d_{p_{5}}$ correspond to ports $p_{1} \cdots p_{5}$ respectively, as they form interactions hosted by $I P_{1}$ (i.e., $a_{1}$ and $a_{2}$ ).

- For each interaction $a \in \gamma_{j}$ that is in external conflict with another interaction, we include an engaged place $e_{a}$ and a free place $f r_{a}$. In Figure 6, only interaction $a_{2}$ is in external conflict, for which we add places $e_{a_{2}}$ and $f r_{a_{2}}$.

Variables and ports. For each port $p$ involved in interactions of $\gamma_{j}$, we include a Boolean variable $x_{p}$. The value of this variable is equal to the value of the same variable in the most recent offer received from the corresponding component. Also, for each component $B_{i}$ involved in interactions of $\gamma_{j}$, we include an integer $n_{i}$ that stores participation number of $B_{i}$. The set of ports of $I P_{j}$ is the following:

- For each component $B_{i}$ involved in interactions of $\gamma_{j}$, we include an offer port $o_{i}$. Each port $o_{i}$ updates the values of variables $n_{i}$ and $x_{p}$ for each port $p$ exported by $B_{i}$. In Figure 6 , ports $o_{1} \cdots o_{4}$ represent offer ports for components $B_{1} \cdots B_{4}$.

- For each port $p$ involved in interactions of $\gamma_{j}$, we include a response port $p$. In Figure 6 , ports $p_{1} \cdots p_{5}$ correspond to the ports that form interactions $a_{1}$ and $a_{2}$.

- For each interaction $a \in \gamma_{j}$ that is in external conflict, we include reservation ports $r_{a}, o k_{a}$, and $f_{a}$. If $a=\left\{p_{i}\right\}_{i \in I}$, the port $r_{a}$ is associated to the variables $\left\{n_{i}\right\}_{i \in I}$, where $I$ is the set of components involved in interaction $a$. In Figure 6, ports $r_{a_{2}}, o k_{a_{2}}$, and $f_{a_{2}}$ represent the external conflict of $a_{2}$ with interactions $a_{3}$ and $a_{4}$.

- For each interaction $a \in \gamma_{j}$ that is not in external conflict, we include a unary port $a$. In Figure 6 , we include unary port $a_{1}$, as $a_{1}$ is only in internal conflict with $a_{2}$.

Transitions. $\quad I P_{j}$ performs two tasks: (1) receiving offers from components in the lower layer and responding to them, and (2) requesting reservation of an interaction from the Reservation Protocol in case of an external conflict. The following set of transitions of $I P_{j}$ performs these two tasks:

- In order to receive offers from a component $B_{i}$, we include transition $\left(w_{i}, o_{i}, r c v_{i}\right)$. If $B_{i}$ participates in an interaction not handled by $I P_{j}$, we also include transition $\left(r c v_{i}, o_{i}, r c v_{i}\right)$ to receive new offers when $B_{i}$ takes part 


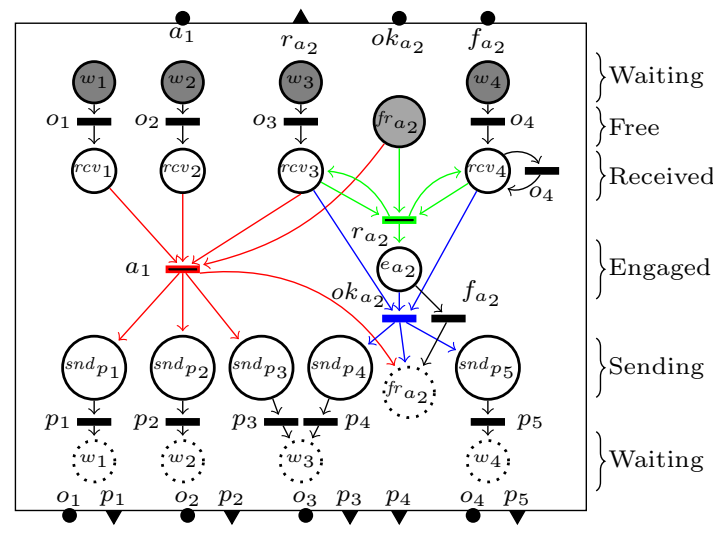

Figure 6: Component $I P_{1}$ in Figure 3.

in such an interaction. Transitions labeled by $o_{1} \cdots o_{4}$ in Figure 6 are of this type.

- Requesting reservation of an interaction $a \in \gamma_{j}$ that is in external conflict is accomplished by transition $\left(\left\{r c v_{i}\right\}_{i \in I} \cup\left\{f r_{a}\right\}, r_{a},\left\{r c v_{i}\right\}_{i \in I} \cup\left\{e_{a}\right\}\right)$, where $I$ is the set of components involved in interaction $a$. This transition is guarded by the predicate $\bigwedge_{i \in I} x_{p_{i}}$ which ensures enabledness of $a$. Notice that this transition is enabled when the token for each participating component is in its corresponding receive place $r c v_{i}$. Execution of this transition results in moving the token from a free place to an engaged place. In Figure 6 , transition $r_{a_{2}}$ is of this type, and is guarded by $x_{p_{4}} \wedge x_{p_{5}}$.

- For the case where the Reservation Protocol responds positively, we include the transition $\left(\left\{r c v_{i}\right\}_{i \in I} \cup\left\{e_{a}\right\}, o k_{a},\left\{s n d_{p_{i}}\right\}_{i \in I} \cup\left\{f r_{a}\right\}\right)$. Upon execution of this transition, the token from the engaged place moves to the free place and the tokens from received move to sending places for informing the corresponding components. Transition $o k_{a_{2}}$ in Figure 6 occurs when interaction $a_{2}$ is successfully reserved by the Reservation Protocol.

- For the case where the Reservation Protocol responds negatively, we include the transition $\left(e_{a}, f_{a}, f r_{a}\right)$. Upon execution of this transition, the token moves from the engaged place to the free place. Transition $f_{a_{2}}$ in Figure 6 occurs when the Reservation Protocol fails to reserve interaction $a_{2}$ for component $I P_{1}$.

- For each interaction $a=\left\{p_{i}\right\}_{i \in I}$ in $\gamma_{j}$ that has only internal conflicts, let $A$ be the set of interactions that are in internal conflict with $a$, but are externally conflicting with other interactions. We include the transition $\left(\left\{r c v_{i}\right\}_{i \in I} \cup\left\{f r_{a^{\prime}}\right\}_{a^{\prime} \in A}, a,\left\{s n d_{p_{i}}\right\}_{i \in I} \cup\left\{f r_{a^{\prime}}\right\}_{a^{\prime} \in A}\right)$. This transition is guarded by the predicate $\bigwedge_{i \in I} x_{p_{i}}$ and moves the tokens from receiving to sending places. Tokens from $f r_{a^{\prime}}$ places ensure that no internally conflicting interaction requested a reservation. The transition labeled by $a_{1}$ in Figure 6 falls in this category.

- Finally, for each component $B_{i}$ exporting $p$, we include the transitions $\left(s n d_{p}, p, w_{i}\right)$. This transition notifies component $B_{i}$ to execute the transition labeled by port $p$. These are transitions labeled by $p_{1} \cdots p_{5}$ in Figure 6 .

\subsection{Reservation Protocol}

As discussed earlier, the main task of the Reservation Protocol is to ensure that externally conflicting interactions are

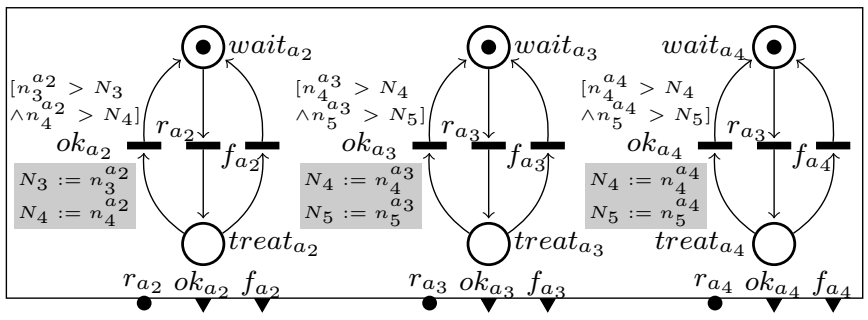

Figure 7: A centralized Reservation Protocol for Figure 3.

executed mutually exclusive. The Reservation Protocol can be implemented using any algorithm that solves the committee coordination problem. Our design of Reservation Protocol allows employing any such algorithm with minimal restrictions.

We adapt a variation of the idea of the message-count technique from [2] as a minimal restriction to ensure that our design makes progress (see Lemma 2) and it does not interfere with exclusion algorithms. This technique is based on counting the number of times that a component interacts. Each component keeps a counter $n$ which indicates the current number of participations of the component in interactions. The Reservation Protocol ensures that each participation number is used only once. That is, each component takes part in only one interaction per transition. To this end, in the Reservation Protocol, for each component $B_{i}$, we keep a variable $N_{i}$ which stores the latest number of participations of $B_{i}$. Whenever a reserve message $r_{a}$ for interaction $a=\left\{p_{i}\right\}_{i \in I}$ is received by the Reservation Protocol, the message provides a set of participation numbers $\left(\left\{n_{i}^{a}\right\}_{i \in I}\right)$ for all components involved in $a$. If for each component $B_{i}$, the participation number $n_{i}^{a}$ is greater than $N_{i}$, then the Reservation Protocol acknowledges successful reservation through port $o k_{a}$ and the participation numbers in the Reservation Protocol are set to values sent by the interaction protocol. To the contrary, if there exists a component whose participation number is less than or equal to what Reservation Protocol has recorded, then the corresponding component has already participated for this number and the Reservation Protocol replies failure via port $f_{a}$.

Now, since the structure and behavior of the Reservation Protocol components depend on the employed algorithm, we only specify an abstract set of minimal restrictions of this layer as follows:

- For each component $B_{i}$, the Reservation Protocol maintains a variable $N_{i}$ indicating the last participation number reserved for $B_{i}$.

- For each interaction $a=\left\{p_{i}\right\}_{i \in I}$ handled by the reservation protocol, we include three ports: $r_{a}, o k_{a}$ and $f_{a}$. The receive-port $r_{a}$ accepts reservation requests containing fresh values of variables $n_{i}^{a}$. The send-ports $o k_{a}$ and $f_{a}$ accept or reject the latest reservation request, and $N_{i}$ variables are updated in case of positive response.

- Each $r_{a}$ message should be acknowledged by exactly one $o k_{a}$ or $f_{a}$ message.

- Each component of the Reservation Protocol should respect the message-count properties described above.

\subsubsection{Centralized Implementation}

Figure 7 shows a centralized Reservation Protocol for the model in Figure 3. In fact, the component in Figure 7 is the component $R P_{1}$ in Figure 3. A reservation request, for instance, $r_{a_{2}}$, contains fresh variables $n_{3}^{a_{2}}$ and $n_{4}^{a_{2}}$ (correspond- 


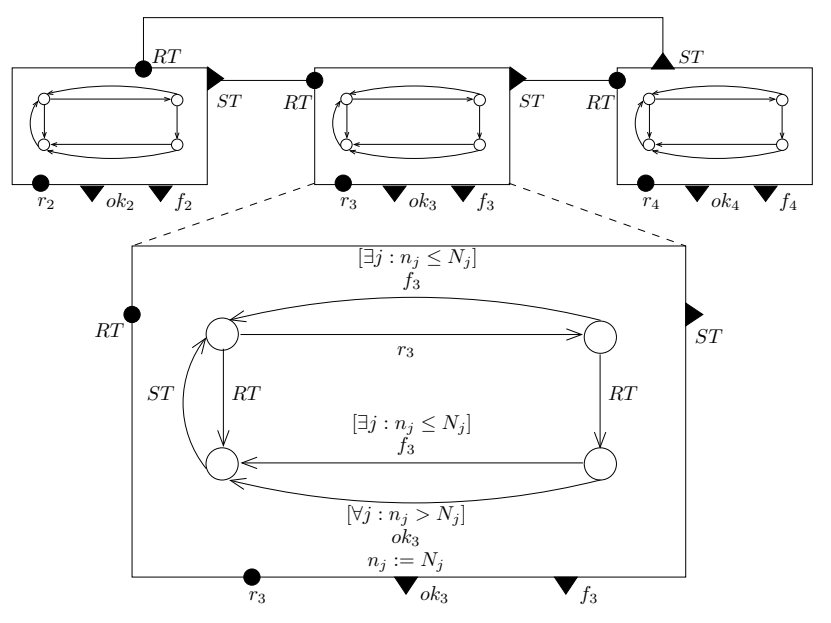

Figure 8: Token-based Reservation Protocol for the BIP models in Figures 1 and 3.

ing to components $B_{3}$ and $\left.B_{4}\right)$. The token representing interaction $a_{2}$ is then moved from place wait $_{a_{2}}$ to place treat $_{a_{2}}$. From this state, the Reservation Protocol can still receive a request for reserving $a_{3}$ and $a_{4}$ since wait $_{a_{3}}$ and wait $_{a_{4}}$ still contain a token. This is where message-counts play their role. The guard of transition $o k_{a_{2}}$ is $\left(n_{3}^{a_{2}}>N_{3}\right) \wedge\left(n_{4}^{a_{2}}>N_{4}\right)$ where $N_{i}$ is the last known used participation number for $B_{i}$. Note that since execution of transitions are atomic in BIP, if transition $o k_{a_{2}}$ is fired, it modifies variables $N_{i}$ atomically (i.e., before any other transition can take place). We denote this implementation by $R P$.

\subsubsection{Token Ring Implementation}

Another example of a Reservation Protocol is inspired by the token-based algorithm due to Bagrodia [1], where we add one reservation component per externally conflicting interaction. Figure 8 shows the respective components for the model presented in Figure 3. Exclusion is ensured using a circulating token carrying $N_{i}$ variables; i.e., the component that owns the token compares the value of the received $n_{i}$ variables with the $N_{i}$ variables from the token. If they are greater, an $o k$ message is sent to the component that handles that interaction and the $N_{i}$ values on the token are updated. Otherwise, a fail message is sent. Subsequently, the reservation component releases the token via port $S T$, which is received by the next component via port $R T$. Obviously, this algorithm allows a better level of distribution at the reservation layer. We denote this implementation by $T R$.

\subsubsection{Implementation Based on Dining Philosophers}

A third choice of Reservation Protocol algorithm is an adaption of the hygienic solution to the dining philosophers problem presented in $[2,5]$. Its Send/Receive BIP implementation is presented in Figure 9. Similar to token ring, each externally conflicting interaction is handled by a separate component. If two interactions are conflicting, the two corresponding components share a fork carrying $N_{i}$ variables corresponding to the atomic components causing the conflict. In order to positively respond to a reserve, a component has to fetch all forks shared with its neighbors. Then, it compares participation numbers received from the reservation request and from the forks and responds accordingly. After such a response, the forks become dirty. Finally, the component sends the forks if it is asked to do so. We denote this implementation by $D P$.

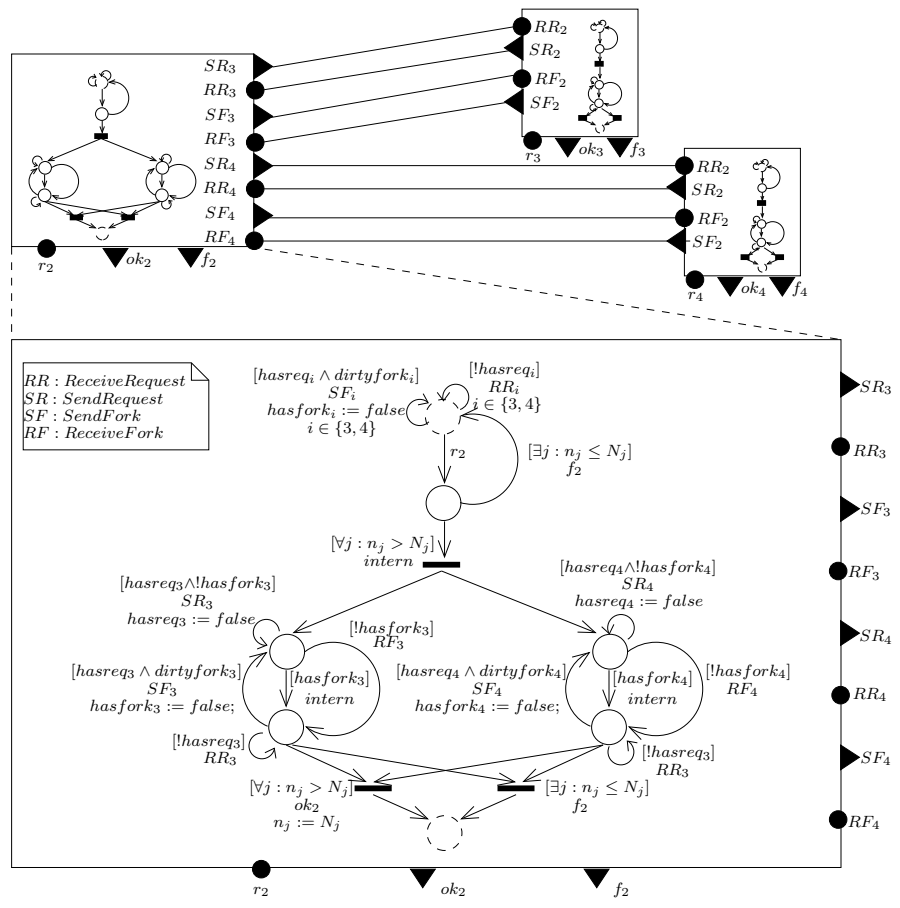

Figure 9: Dining philosophers-based Reservation Protocol for the BIP models in Figures 1 and 3.

\subsection{Cross-Layer Interactions}

In this subsection, we define the interactions of our 3-layer model. Following Definition 4, we construct Send/Receive interactions by specifying which one is the sender. Given a BIP model $\gamma\left(B_{1} \cdots B_{n}\right)$, a partition $\gamma_{1} \cdots \gamma_{m}$, and the obtained Send/Receive components $B_{1}^{S R} \cdots B_{n}^{S R}$, interaction protocol components $I P_{1} \cdots I P_{m}$, and Reservation Protocol components $R P_{1} \cdots R P_{k}$, we construct the Send/Receive interactions $\gamma^{S R}$ according to Definition 4 as follows:

- For each component $B_{i}, \gamma^{S R}$ contains a multicast connector formed by all ports $o_{i}$, where $B_{i}$ is the sender.

- For each Interaction Protocol component $I P_{j}$ and port $p$ in $I P_{j}$, we include a binary interaction, such that port $p$ of $I P_{j}$ is the sender, and, port $p$ of the corresponding component in the components layer is the receiver.

- For each interaction $a$ that is in external conflict, $\gamma^{S R}$ contains an interaction between $r_{a}$ ports, such that the Interaction Protocol is the sender and Reservation Protocol is the receiver. Likewise, $\gamma^{S R}$ contains interactions between $o k_{a}$ and $f_{a}$ ports.

Note that the interaction do not depend on the Reservation Protocol. The entire model obtained is denoted $B_{R P}^{S R}, B_{T R}^{S R}$ or $B_{D P}^{S R}$ following the embedded Reservation Protocol. The interactions between the three layers of our running example are presented in Figure 3. The send-ports are graphically denoted by triangles and receive-ports by bullets.

\section{CORRECTNESS}

In Subsection 5.1, we show that our 3-layer model meets the constraints of the Send/Receive model specified in Section 3. In Subsection 5.2, we prove that a BIP model is observationally equivalent with the BIP model obtained by the transformation of Section 4. Finally, we prove the correctness of 
models embedding different implementations of Reservation Protocol in Subsection $5.3^{1}$.

\subsection{Compliance with Send-Receive Models}

Proposition 1 Given a BIP model B, the model $B^{S R}$ obtained by transformation of Section 4 meets the constraints of Definition 4.

Proof sketch. We need to show that receive-ports of $B^{S R}$ are enabled whenever the corresponding send-ports are enabled. This holds since communications between two successive layers follow a request/acknowledgement pattern. Whenever a layer sends a request, it enables the receive-port for receiving acknowledgement and no new request is sent until the first one is acknowledged.

\subsection{Observational Equivalence between Origi- nal and Transformed BIP Models}

We recall the definition of observational equivalence of two transition systems $A=\left(Q_{A}, P \cup\{\beta\}, \rightarrow_{A}\right)$ and $B=\left(Q_{B}, P \cup\right.$ $\left.\{\beta\}, \rightarrow_{B}\right)$. It is based on the usual definition of weak bisimilarity [7], where $\beta$-transitions are considered unobservable. The same definition is trivially extended for atomic and composite BIP components.

Definition 7 (Weak Simulation) $A$ weak simulation over $A$ and $B$, denoted $A \subset B$, is a relation $R \subseteq Q_{A} \times Q_{B}$, such that we have $\forall(q, r) \in R, a \in P: q \stackrel{a}{\rightarrow}_{A} q^{\prime} \Longrightarrow \exists r^{\prime}$ : $\left(q^{\prime}, r^{\prime}\right) \in R \wedge r{\stackrel{\beta^{*} a \beta^{*}}{\rightarrow}}_{B} r^{\prime}$ and $\forall(q, r) \in R: q \stackrel{\beta}{\rightarrow}_{A} q^{\prime} \Longrightarrow \exists r^{\prime}:$ $\left(q^{\prime}, r^{\prime}\right) \in R \wedge r{\stackrel{\beta^{*}}{\rightarrow} B}_{B} r^{\prime}$

A weak bisimulation over $A$ and $B$ is a relation $R$ such that $R$ and $R^{-1}$ are both weak simulations. We say that $A$ and $B$ are observationally equivalent and we write $A \sim B$ if for each state of $A$ there is a weakly bisimilar state of $B$ and conversely. In this subsection, our goal is to show that $B$ and $B^{S R}$ are observationally equivalent. We consider the correspondence betwen actions of $B$ and $B^{S R}$ as follows. For each interaction $a \in \gamma$, where $\gamma$ is the set of interactions of $B$, we associate either the binary interaction $o k_{a}$ or the unary interaction $a$, depending upon existence of an external conflict. All other interactions (offer, response, reserve, fail) are unobservable and denoted $\beta$.

We proceed as follows to complete the proof of observational equivalence. Amongst unobservable actions $\beta$, we distinguish between $\beta_{1}$ actions, that are communication interactions between the components layer and the Interaction Protocol (namely offer and response), and $\beta_{2}$ actions that are communications between the Interaction Protocol and and Reservation Protocol (namely reserve and fail). We denote $q^{S R}$ a state of $B^{S R}$ and $q$ a state of $B$. A state of $B^{S R}$ from where no $\beta_{1}$ action is possible is called a stable state, in the sense that any $\beta$ action from this state does not change the state of the component layer.

Lemma 1 From any state $q^{S R}$, there exists a unique stable state $[q]^{S R}$ such that $q^{S R} \stackrel{\beta_{1}^{*}}{\longrightarrow}[q]^{S R}$.

We now show a property of the participation numbers. Let $B . n$ mean 'the variable $n$ that belongs to component $B$ '.

\footnotetext{
${ }^{1}$ For reasons of space, all proofs are available at http:// www-verimag.imag. fr/ $\sim$ bonakdar/emsoft 10 . pdf.
}

Lemma 2 When $B^{S R}$ is in a stable state, for each couple $(i, j)$, such that $B_{i}$ is involved in interactions handled by $I P_{j}$, we have $B_{i} . n_{i}=I P_{j} . n_{i}>R P . N_{i}$.

Since we need to take into account participation numbers $n_{i}$, we introduce an intermediate centralized model $B^{n}$. This new model is a copy of $B$ that includes in each atomic component an additional variable $n_{i}$ which is incremented whenever a transition is executed. As $B$ and $B^{n}$ have identical set of states and transitions labeled by the same ports, they are observationally equivalent. (They are even strongly bisimilar.)

\section{Lemma $3 B \sim B^{n}$.}

We are now ready to state and prove our central result.

Proposition $2 B^{S R} \sim B^{n}$.

Proof sketch. We define a relation $R$ between the states $Q^{S R}$ of $B^{S R}$ and the states $Q$ of $B^{n}$ as follows: $R=\left\{\left(q^{S R}, q\right) \mid \forall i \in\right.$ $\left.I:[q]_{i}^{S R}=q_{i}\right\}$ where $q_{i}$ denotes the state of $B_{i}^{n}$ at global state $q$ and $[q]_{i}^{S R}$ denotes the state of $B_{i}^{S R}$ at global state $[q]^{S R}$. The three next assertions hold and prove that $R$ is a weak bisimulation:

(i) If $\left(q^{S R}, q\right) \in R$ and $q^{S R} \stackrel{\beta}{\longrightarrow} r^{S R}$, then $\left(r^{S R}, q\right) \in R$.

(ii) If $\left(q^{S R}, q\right) \in R$ and $q^{S R} \stackrel{a}{\longrightarrow} r^{S R}$, then $\exists r \in Q: q \stackrel{a}{\longrightarrow} r$ and $\left(r^{S R}, r\right) \in R$.

(iii) If $\left(q^{S R}, q\right) \in \underset{S R}{\in}$ and $q \stackrel{a}{\longrightarrow} r$, then $\exists r^{S R} \in Q^{S R}: q^{S R} \stackrel{\beta^{*} a}{\longrightarrow}$ $r^{S R}$ and $\left(r^{S R}, r\right) \in R$.

\subsection{Interoperability of Reservation Protocol}

As mentioned in Subsection 4.3, the centralized implementation $R P$ of the Reservation Protocol can be seen as a specification. We also proposed two other implementations, respectively, token-ring $T R$ and dining philosophers $D P$. However, these implementations are not observationally equivalent to the centralized implementation. More precisely, the centralized version defines the most liberal implementation: if two reservation requests $a_{1}$ and $a_{2}$ are received, the protocol may or may not acknowledge them, in a specific order. This general behavior is not implemented neither by the token ring nor by the dining philosophers implementations. In the case of token ring, the response may depend on the order the token travels through the components. In the case of dining philosophers, the order may depend on places and the current status of forks.

Nevertheless, we can prove an observational equivalence if we consider weaker versions of the above implementations. More precisely, for the token ring protocol, consider the weaker version $T R^{(w)}$ which allows to release the token or provide a fail answer regardless of the values of counters. Likewise, for the dining philosophers protocol, consider the weaker version $D P^{(w)}$, where forks can always be sent to neighbors, regardless of their status and the values of counters. Clearly, a weakened Reservation Protocol is not desirable for a concrete implementation since they do not enforce progress. But, they play a technical role in proving the correctness of our approach. The following proposition establishes the relation between the different implementations of the Reservation Protocol.

Proposition 3 (i) $R P \sim T R^{(w)} \sim D P^{(w)}$

(ii) $T R \subset T R^{(w)}, D P \subset D P^{(w)}$. 


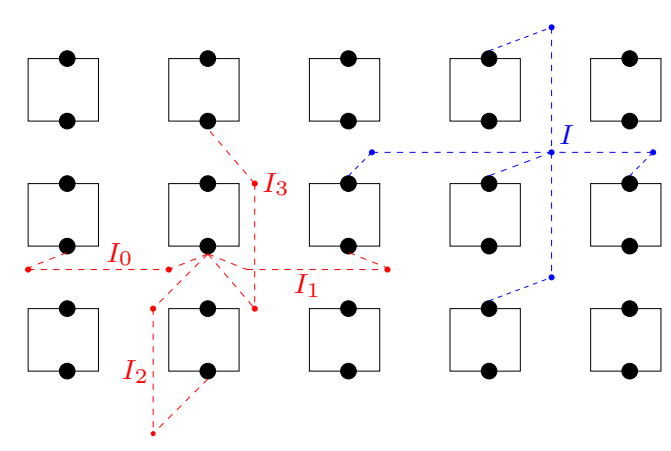

Figure 10: Partial BIP model for diffusing computations.

Let us denote by $B_{X}^{S R}$ the 3-layer model obtained from the initial system $B$ and embedding algorithm $X$ in the Reservation Protocol. Also, let us denote $\operatorname{Tr}(B)$ the set of all possible traces of observable actions allowed by an execution of $B$. The following proposition states the correctness of our implementation.

Proposition 4 (i) $B \sim B^{S R} \sim B_{T R^{(w)}}^{S R} \sim B_{D P(w)}^{S R}$ (ii) $\operatorname{Tr}(B) \supseteq \operatorname{Tr}\left(B_{T R}^{S R}\right)$ and $\operatorname{Tr}(B) \supseteq \operatorname{Tr}\left(B_{D P}^{S R}\right)$.

\section{EXPERIMENTAL RESULTS}

In this section, we present the results of our experiments. Our implementation automatically generates $\mathrm{C}++$ code from the 3-layer BIP model developed in Sections 3 and 4, where Send/Receive interactions are implemented by TCP sockets primitives. Code generation involves generating stand-alone executables for each Send/Receive component in each layer of the 3-layer BIP model. The code of each component simulates its automaton or Petri net using the technique presented in [4].

In the following, we denote each experiment scenario by $(i, X)$, where $i$ is the number of interaction partitions and $X$ is the choice among the three Reservation Protocols described in Subsection 4.3 (i.e., $R P$, $T R$, or $D P$ ). For the case where partitioning of interactions results in having no external conflicts and, hence, requiring no reservation component, we use the symbol ' - ' to denote an empty Reservation Protocol. All experiments in this section are conducted on five quad-Xeon $2.6 \mathrm{GHz}$ machines with 6GB RAM running under Debian Linux and connected via a $100 \mathrm{Mbps}$ Ethernet network. Our aim is to show that different conflict resolution algorithms and partitioning may result in significantly different performance.

For our experiments, we model a simplified version of Dijkstra-Scholten termination detection algorithm for diffusing computations [6] in BIP. Diffusing computation is the task of propagating a message across a distributed system; i.e., a wave that starts from an initial node and diffuse to all processes in a distributed system. Diffusing computation has numerous applications such as traditional distributed deadlock detection and reprogramming of modern sensor networks. One challenge in diffusing computation is to detect its termination. In our version, we consider a torus (wrapped around grid) topology for a set of distributed processes, where a spanning tree throughout the distributed system already exists; each process has a unique parent and the root process is its own parent. Termination detection is achieved in two phases: (1) the root of the spanning tree possesses a message and initiates a propagation wave, so that each process sends the

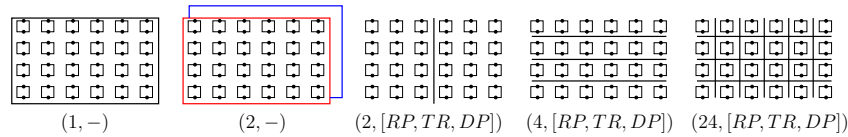

Figure 11: Different scenarios for diffusing computations.

message to its children, and (2) once the first wave of messages reaches the leaves of the tree, a completion wave starts, where a parent is complete once all its children are complete. In this setting, when the root is complete, termination is detected.

Our BIP model has $n \times m$ atomic components (see Figure 10 for a partial model). Each component participates in two types of interactions: (1) four binary rendezvous interactions (e.g., $\left.I_{0} \cdots I_{3}\right)$ to propagate the message to its children (as in a torus topology, each node has four neighbors and, hence, potentially four children), and (2) one 5-ary rendezvous interaction (e.g., $I$ ) for the completion wave, as each parent has to wait for all its children to complete.

Our first set of experiments is on a $6 \times 4$ torus. We apply different partitioning scenarios as illustrated in Figure 11. Figure 12(a) shows the time needed for 100 rounds of detecting termination of diffusing communication for each scenario. In the first two scenarios, the interactions are partitioned, so that all conflicts are internal and, hence, resolved locally by the Interaction Protocol. In case of $(2,-)$, all interactions of the propagation wave are grouped into one component of the Interaction Protocol and all interactions related to the completion wave are grouped into the second component. Such grouping does not allow parallel execution of interactions. This is the main reason that the performance of $(1,-)$ and $(2,-)$ are the worst in Figure 12(a).

Next, we group all interactions involved in components $1 \cdots 12$ into one component and the rest in a second component of the Interaction Protocol. This constitutes experiments $(2, R P),(2, T R)$, and $(2, D P)$. Such partitioning allows more parallelism during propagation and completion waves, as an interaction in the first partition can be executed in parallel with an interaction in the second partition ${ }^{2}$. This is why the performance of $(2, R P / T R / D P)$ is better than $(1,-)$ and $(2,-)$. Now, since almost all propagation interactions conflict with each other and so do all completion interactions, in case of the dining philosophers algorithm, the conflict graph is not dense. Hence, a small of number decisions can be made in a local neighborhood of philosophers. It follows that the performance of $(2, T R)$ is quite competitive with $(2, D P)$. It can also be seen that $(2, R P)$ performs as good as $(2, T R)$ and $(2, D P)$. This is due to the fact that there exist only two partitions, which results in a low number of reservation requests.

Figure 12(a) also shows the same type of experiments with 4 and 24 partitions. Similar to the case of two partitions, the performace of $T R$ and $R P$ for 4 and 24 partitions compete with each other. However, $R P$ and $T R$ outperform $D P$. This is due to the fact that in case of $D P$, each philosopher needs to acquire 4 forks, which requires considerable communication. On the other hand, $T R$ does not require as much communication, as the only task it has to do is releasing and acquiring the token. Moreover, the level of parallelism in DP in case of a $6 \times 4$ torus is not high enough to overcome the communication volume.

\footnotetext{
${ }^{2}$ Execution of each interaction involves $10 \mathrm{~ms}$ suspension of the corresponding component in the Interaction Protocol to perform and $\mathrm{I} / \mathrm{O}$ command.
} 


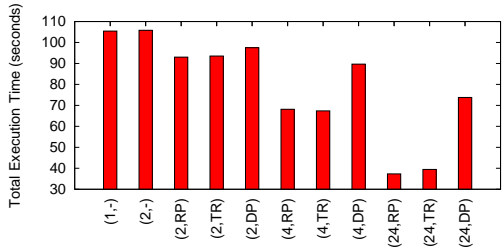

(a) Torus $6 \times 4$

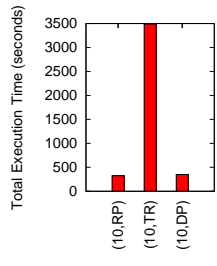

(b) Torus $20 \times 20$
Figure 12: Performance of termination detection in diffusing computation in different scenarios.

In the next experiment, following the lesson learned from the tradeoff between communication volume and parallelism, we design a scenario where we exploit the fact that each reservation component in $D P$ resolves conflict through communicating with its neighboring components. This is not the case in $T R$. Thus, we consider a $20 \times 20$ torus. As can be seen in Figure 12(b), the performance of $D P$ is significantly better than $T R$. This is solely because when we have a large number of components, in $T R$, the token has to travel a long way in order to allow parallel execution of interactions. To the contrary, in $D P$, the Reservation Protocol components act in their local neighborhood and although more communication is needed, it allows better concurrency and, hence, higher simultaneous execution of interactions. We expect that by increasing the size of the torus, DP outperforms $R P$ as well.

We conclude this section by stating the main lesson learned from our experiments:

Different partitioning schemes and choice of committee coordination algorithm for distributed conflict resolution suit different topologies and settings although they serve a common purpose. Designers of distributed applications should have access to a library of algorithms and choose the best according to parameters of the application.

\section{CONCLUSION}

We focused on developing a generic framework for automated transformation of high-level BIP models in terms of a set of components glued by rendezvous interactions into distributed implementations. In a distributed setting, implementation of a multi-party rendezvous results in solving the committee coordination problem [5], where a set of professors are organized in a set of committees and two committees can meet concurrently only if they have no professor in common; i.e., they are not conflicting. Our transformation consists of two steps. First, it takes as input a BIP model and generates another BIP model which contains components glued by Send/Receive interactions in the following three layers: (1) the components layer consists of a transformation of behavioral atomic components in the original model, (2) the Interaction Protocol detects enabledness of interactions of the original model and executes them after resolving conflicts either locally or by the help of the third layer, and (3) the Reservation Protocol resolves conflicts in a distributed fashion. The Reservation Protocol implements a committee coordination algorithm and our design allows employing any such algorithm. The second step of our transformation takes the intermediate three-layer BIP model as input and generates $\mathrm{C}++$ executables using TCP sockets for communication. We reported the lessons learned through conducting several experiments using different algorithms in the Reservation Protocol and partitioning schemes. As predicated, there is no silver bullet to automate code generation of distributed applications. Hence, designers must have access to a formal framework and a rich library of algorithms such as the ones presented in this paper to develop correct and yet efficient distributed applications automatically.

For future work, we are considering several research directions. An important extension is to allow the Reservation Protocol to incorporate different algorithms for conflict resolution simultaneously, so that each set of conflicting interactions within the same system is handled by the most appropriate algorithm. In this context, we are also planning to explore other algorithms, such as solutions to distributed graph matching and distributed independent set for better understanding of tradeoffs between parallelism, load balancing, and network traffic. Another important line of research is to study the overhead of our transformation where communication cost is crucial such as in peer-to-peer and large sensor networks. Finally, given the recent advances in the multicore technology, we plan to customize our transformation for multi-core platforms.

\section{REFERENCES}

[1] R. Bagrodia. A distributed algorithm to implement n-party rendevouz. In Foundations of Software Technology and Theoretical Computer Science, Seventh Conference (FSTTCS), pages 138-152, 1987.

[2] R. Bagrodia. Process synchronization: Design and performance evaluation of distributed algorithms. IEEE Transactions on Software Engineering (TSE), 15(9):1053-1065, 1989.

[3] A. Basu, P. Bidinger, M. Bozga, and J. Sifakis. Distributed semantics and implementation for systems with interaction and priority. In Formal Techniques for Networked and Distributed Systems (FORTE), pages 116-133, 2008.

[4] B. Bonakdarpour, M. Bozga, M. Jaber, J. Quilbeauf, and J. Sifakis. From high-level component-based models to distributed implementations. Technical Report TR-2010-9, VERIMAG, March 2010.

[5] K. M. Chandy and J. Misra. Parallel program design: a foundation. Addison-Wesley Longman Publishing Co., Inc., Boston, MA, USA, 1988.

6] E. W. Dijkstra and C. S. Scholten. Termination detection for diffusing computations. Information Processing Letters, 11(1):1-4, 1980.

[7] R. Milner. Communication and concurrency. Prentice Hall International (UK) Ltd., Hertfordshire, UK, 1995. 